\title{
Zur quantitativen dünnschichtchromatographischen Bestimmung freier Gallensäuren
}

\author{
Von \\ B. FrosCh und H. WAGENER \\ Aus der II. Medizinischen Klinik und Poliklinik der Freien Universität Berlin (Direktor: Prof. Dr. G. Schettler)
}

(Der Schriftleitung zugegangen am 18. Mai 1963)

\begin{abstract}
Es wird eine Methode zur quantitativen Bestimmung der dünnschichtchromatographisch unvollständig zu trennenden Desoxycholsäure und Chenodesoxycholsäure mittels Absorptionsspektrophotometrie beschrieben.

Deoxycholic and chenodeoxycholic acid, which can only be partially separated by thin layer chromatography, are determined quantitatively by absorption spectrophotometry.
\end{abstract}

Mit den bisher bekannten Verfahren zur Dünnschichtchromatographie freier Gallensäuren wird eine nur ungenügende Trennung der beiden Dihydroxycholansäuren $3 \alpha$-12 $\alpha$-Desoxycholansäure (Desoxycholsäure $={ } \mathrm{DC}(\mathrm{C})$ und $3 \alpha-7 \alpha$-Desoxycholansäure (Chenodesoxycholsäure $\left.={ } \mathrm{CDC}^{c 6}\right)$ erzielt $(1,2,3)$. Wenn auch mittels der von HAMimion (4) und ENEROTH (5) angegebenen Lösungsmittelsysteme eine bessere Trennung der beiden Isomere erhalten wird, bleiben die Abstände zwischen den beiden Flecken der DC und $\mathrm{CDC}$ gering. Für eine quantitative Elution sind jedoch größere $R_{\mathrm{F}}$-Wert-Unterschiede unerläßlich, falls nicht der direkte Nachweis durch solche Sprühreagenzien möglich ist, welche die nachfolgende quantitative Bestimmung nicht stören. Da bisher keine Färbeverfahren zur Lokalisation der Flecke auf det Dünnschichtplatte bekannt sind, welche die nachfolgende Reaktion der Gallensäure mit Schwefelsäure nicht stören, erfolgt die Lokalisation der erhaltenen Flecke zweckmäßig mit sog. Leitchromatogrammen. Gerade dieses Vorgehen macht größtmögliche $R_{\mathrm{F}}$-Wert-Unterschiede erforderlich, da häufig trotż sorgfältiger Einhaltung der Versuchsbedingungen Schwankungen der $R_{\mathrm{F}}$-Werte und damit $\mathrm{z}$. B. elongierte Flecke auftreten. Wir hielten es deshalb für zweckmäßig, die Desoxycholsäure und Chenodesoxycholsäure gemeinsam zu erfassen und eine Methode zu entwickeln, die quantitative Aussagen über den Anteil jeder einzelnen Dihydroxycholansäure gestattet. Dazu bedienten wir uns der Beobachtung, daß Dihydroxycholansäuregemische verschiedener Zusammensetzung für das. betreffende Gemisch charakteristische Absorptionskurven ergeben.

\section{Methodik}

1. Reagenzien: Kieselgel $\mathrm{G}$ für Dünnschichtchromatographie, Fa. Merck; Essigsäure p. a.; Toluol p. a.; Schwefelsäure $65 \%$ (g/g); Phosphormolybdänsäure, 5 proz. äthanol. Lösung.

2. Geräte: Ausrïstung zur Dünnschichtchromatographie der Fa. Desaga, Heidelberg. Agla-Mikrometerspritze der Fa. BourroughWelcome \& Co., London. Spektralphotometer PMQ II der Fa. C. Zeiss, Oberkochen.
3. Gallensäuren: Cholsäure, Desoxycholsäure und Chenodesoxycholsäure (Fa. California Corporation for Biochemical Research, Los Angeles). $\mathrm{Da}$ diese Handelspräparate $z$. T. erhebliche Verunreinigungen enthielten, wurden sie säulenchromatographisch mittels der Phasenumkehr-Chromatographie nach Norman (6) bzw. GäNSHIRT und Mitarbeitern (1) gereinigt. Das Eluat wurde dabei in $5 \mathrm{~m} /$-Fraktionen gesammelt und diese dünnschichtchromatographisch analysiert. Entsprechende Fraktionen wurden vereinigt und zum Teil nach dem Eindampfen nochmals umkristallisiert. Auf diese Weise wurden reine Chol- und Desoxycholsäure erhalten. Das Chenodesoxycholsäure-Präparat dagegen enthielt noch eine minimale, nur beim Auftragen größerer Mengen nachweisbare unbekannte Verunreinigung.

4. Dünnschichtchromatographie und spektrophotometrische Bestimmung: Die Herstellung der mit Kieselgel beschichteten Platten erfolgte nach STAHL; allerdings bewährte sich uns ein Mischungsverhältnis von $30 \mathrm{~g}$ Kieselgel und $55 \mathrm{ml}$ Aqua dest. Die lufttrockenen Platten wurden eine Std. bei $100^{\circ}$ getrocknet und offen aufbewahrt. Als Lösungsmittelsysteme verwendeten wir Toluol: Eisessig: Wasser = 5:5:1 bzw. Toluol: Eisessig: Wasser $=7,5: 12,5: 1$. Die Laufstrecke für das erste System betrug 12, für das zweite $16 \mathrm{~cm}$. Die Substanzen wurden in äthanol. Lösungen mittels Agla-Mikrometerspritze punktförmig $2 \mathrm{~cm}$ über dem unteren Plattentand aufgetragen. Die Laufzeiten betrugen in der gesättigten Kammer bei $18^{\circ} 2-3 \mathrm{Stdn}$. Nach dem Abdunsten des Lösungsmittels wurden an beiden Seiten der Platten durch Besprühen mit Phosphormolybdänsäurereagenz und Erwärmen auf $70^{\circ}$ Leitchromatogramme entwickelt und aus der unbesprühten Plattenmitte die entsprechenden Kieselgelbezirke abgeschabt. Diese wurden in Zentrifugengläschen gebracht, mit genau $3 \mathrm{ml} 65 \%(\mathrm{~g} / \mathrm{g})$ Schwefelsäure versetzt und $60 \mathrm{Min}$. bei $60^{\circ} \mathrm{im}$ Wasserbad erhitzt. Danach wurden die Gläschen $15 \mathrm{Min}$. bei $3000 \mathrm{U} / \mathrm{Min}$. zentrifugiert und der klare Uberstand zur photometrischen Bestimmung in $1 \mathrm{~cm}$-Küvetten dekantiert. Die Ablesung erfolgte gegen Leerwerte, die aus flächengleichen, unbeladenen Schichtbezirken entsprechender $R_{\mathrm{F}}$ Werte von jeder Platte bei gleicher Behandlung angesetzt wurden. Von jeder Probe wurde eine Absorptionskurve zwischen 370 und $395 \mathrm{~m} \mu$ aufgenommen.

\section{Ergebnisse}

Zur Herstellung von Eichkurven wurden von Cholsäure, Desoxycholsäure und Chenodesoxycholsäure Mengen zwischen 10 und $80 \mu \mathrm{g}$ aufgetragen und ihre Extinktion nach der oben beschriebenen Methode bei konstanter Wellenlänge $(\lambda=385 \mathrm{~m} \mu)$ ermittelt. Für die untersuchten Gallensäuren ergibt sich eine lineare Beziehung $z$ wischen Extinktion und aufgetragener Menge. 


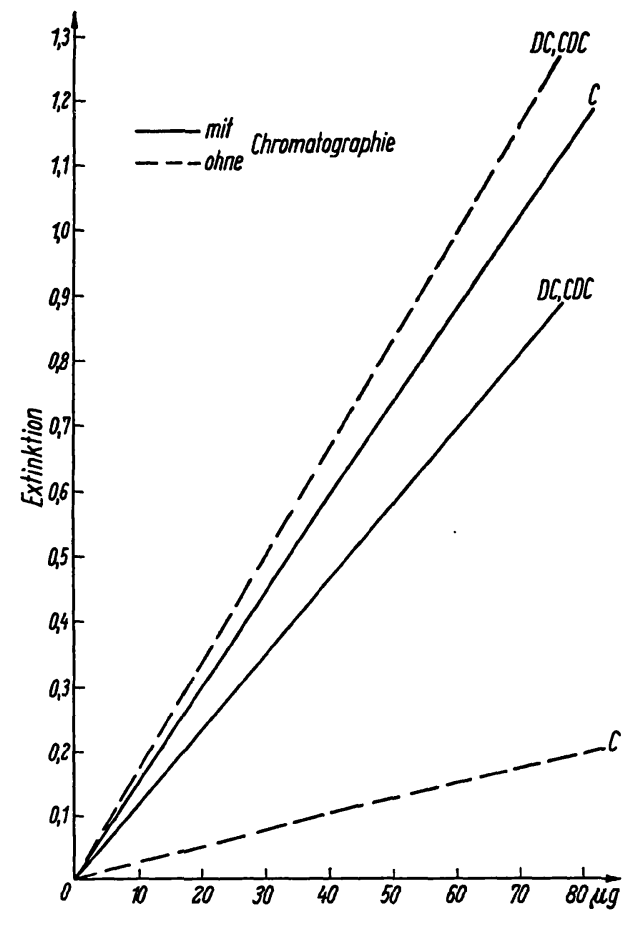

Abb. 1

Eichkurven für Cholsäure (C), Desoxycholsäure (DC) und Chenodesoxycholsäure (CDC) direkt und nach Dünnschichtchromatographie. Ordinate: Extinktion bei $385 \mathrm{~m} \mu$, Abszisse: eingesetzte Gallensäuremengen in $\mu \mathrm{g}$.

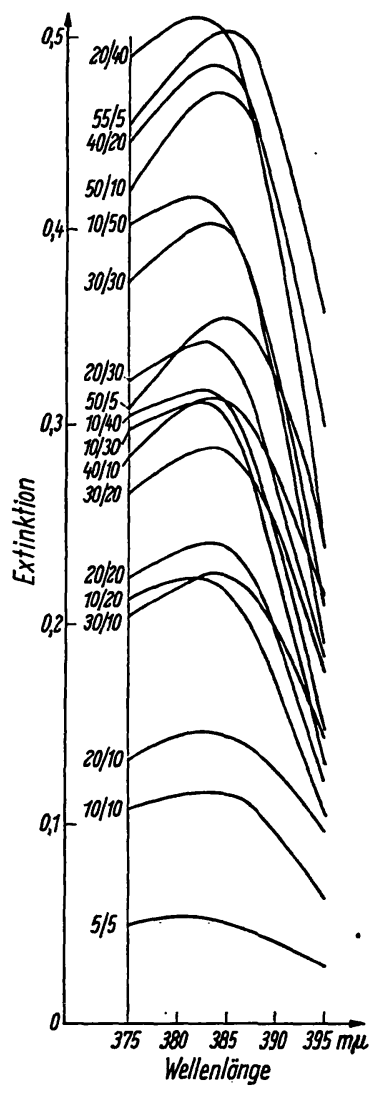

Abb. 2

Absorptionskurven verschiedener Desoxycholsäure/Chenodesoxycholsäure-Gemische zwischen 375 und $395 \mathrm{~m} \mu$.
Wie aus Abbildung 1 ersichtlich, haben Desoxycholsäure und Chenodesoxycholsäure bei $385 \mathrm{~m} \mu$ identische Eichkurven, während die der Cholsäure einen steileren Anstieg aufweist. Auffällig ist, daß die aus nicht chromatographierten Testmengen erhaltenen Eichkurven sich umgekehrt verhalten, da $B$ also die Eichkurve für Cholsäure den geringeren Anstieg aufweist. Der Cholsäuregehalt einer Lösung läßt sich durch Ablesung aus dieser Eichkurve leicht ermitteln. Die Eichkurven für Desoxy- und Chenodesoxycholsäure dagegen können nicht angewandt werden, da diese beiden Gallensäuren dünnschichtchromatographisch für eine quantitative Bestimmung nicht ausreichend getrennt werden. Zwar läßt sich von den Dihydroxycholansäuren eine gemeinsame Extinktion bestimmen, jedoch kann aus dieser nicht auf die Anteile der beiden Säuren geschlossen werden. Eine Extinktion von z. B. 0,155 (Tabelle 1)

Tab. 1

Extinktionen bei $385 \mathrm{~m} \mu$

\begin{tabular}{lccc}
\hline & $10 \mu \mathrm{g}$ & $20 \mu \mathrm{g}$ & $30 \mu \mathrm{g}$ \\
\hline $\mathrm{E}_{385} \mathrm{~m} \mu \mathrm{DC}$ & 0,155 & 0,320 & 0,505 \\
$\mathrm{E}_{385} \mathrm{~m} \mu \mathrm{CDC}$ & 0,155 & 0,232 & 0,500 \\
$\mathrm{E}_{385} \mathrm{~m} \mu \mathrm{DC}+\mathrm{CDC}$ gemessen & 0,115 & 0,238 & 0,400 \\
$\begin{array}{l}\mathrm{E}_{385} \mathrm{~m} \mu \mathrm{DC}+\mathrm{CDC} \text { als Summe } \\
\text { berechnet }\end{array}$ & 0,310 & 0,643 & 1,005 \\
\hline
\end{tabular}

entspricht zwar $10 \mu \mathrm{g}$ Desoxycholsäure oder auch $10 \mu \mathrm{g}$ Chenodesoxycholsäure, erlaubt aber keine Aussage über die Zusammensetzung des tatsächlich vorliegenden Gemisches. Vergleicht man nämlich die Extinktion gleicher Mengen von Desoxycholsäure und Chenodesoxycholsäure mit der verschiedener aus gleichen Mengen gebildeter Gemische der beiden Dihydroxycholansäùren (s. Tabelle 1), so ergibt sịch, daß die Extinktion des Gemisches beider Gallensäuren nicht etwa die Summe der einzelnen Extinktionen darstellt. - Um dennoch zu einer quantitativen Aussage über die vorliegenden Mengen der beiden chromatographisch ungenügend getrennten Dihydroxycholansäuren zu kommen, haben wir ihre Absorptionskurven aufgenommen. Die Anteile von Desoxycholsäure und Chenodesoxycholsäure wählten wir so, $\mathrm{da} ß$ beide Gallensäuren zusammen nicht mehr als $60 \mu \mathrm{g}$ ausmachen, zwischen 5 und $60 \mu \mathrm{g}$ jedoch in $5 \mu \mathrm{g}$-Differenzen alle Kombinationen von Desoxy- und Chenodesoxycholsäure gemessen werden. Die 66 Kombinationen der beiden Gallensäuren wurden aus frisch bereiteten äthanol. Lösungen der Reinsubstanzen mittels AglaMikrometerspritze zusammen punktförmig aufgetragen und nach dem oben beschriebenen Verfahren entwickelt. Die Extinktionswerte jedes Gemisches wurden 4-6mal gemessen. Für die Darstellung der Absorptionskurven wurden die Mittelwerte benutzt. Einen Teil der Absorptionskurven zeigt Abbildung 2. Wir haben zugunsten einer besseren Übersicht darauf verzichtet, alle 66 Kurven darzustellen. Es muß jedoch 
darauf hingewiesen werden, daß trotz der großen Zahl von Absorptionskurven keine identischen Kurvenpaare gefunden wurden, vielmehr alle Kurven charakteristische Merkmale aufweisen, die ihre Zuordnung zu aufgetragenen Substanzgemischen bekannter Zusammensetzung erlauben. Die einzelnen Absorptionskurven sind charakterisiert durch die Lage des Absorptionsmaximums, seinen Extinktionswert und durch den Anstieg zwischen $375 \mathrm{~m} \mu$ und der Wellenlänge des Maximums.

Voraussetzung für die Brauchbarkeit der beschriebenen Absorptionskurven zur Ermittlung der Zusammensetzung eines unbekannten Dihydroxycholansäuregemisches ist die Reproduzierbarkeit der Extinktionswerte. $\mathrm{Zu}$ diesem Zweck analysierten wir verschiedene Gemische von Desoxychol- und Chenodesoxycholsäure mehrfach. Als Beispiel zeigt Tabelle 2 die Mittelwerte

Tab. 2

Mittelwerte und Standardabweichungen der Extinktionen eines Gemisches von $30 \mu \mathrm{g} \mathrm{DC}$ und $25 \mu \mathrm{g} C D C$ aus 10 Messungen berechnet

\begin{tabular}{cccc}
\hline $\begin{array}{c}\text { Wellenlänge } \\
(\mathrm{m} \mu)\end{array}$ & $\begin{array}{c}\text { Extinktion } \\
\text { (Mittelwert) }\end{array}$ & $\begin{array}{c}\text { Standardab- } \\
\text { weichung士 }\end{array}$ & $\begin{array}{c}\text { Standardab- } \\
\text { weichung in } \\
\text { 土o des Mit- } \\
\text { telwertes }\end{array}$ \\
\hline 375 & 0,301 & 0,0041 & 1,23 \\
380 & 0,321 & 0,0041 & 1,23 \\
381 & 0,324 & 0,0036 & 1,16 \\
382 & 0,326 & 0,0039 & 1,27 \\
383 & 0,328 & 0,0042 & 1,38 \\
384 & 0,328 & 0,0041 & 1,34 \\
385 & 0,327 & 0,0041 & 1,34 \\
386 & 0,324 & 0,0050 & 1,62 \\
387 & 0,318 & 0,0054 & 1,72 \\
388 & 0,309 & 0,0064 & 1,87 \\
389 & 0,298 & 0,0076 & 2,26 \\
390 & 0,282 & 0,0076 & 2,14 \\
\hline
\end{tabular}

und Standardabweichungen eines Gemisches aus $30 \mu \mathrm{g}$ Desoxycholsäure und $25 \mu \mathrm{g}$ Chenodesoxycholsäure, welches $10 \mathrm{mal}$ dem gesamten Arbeitsgang unterworfen wurde. Die somit für jede gemessene Wellenlänge aus 10 abgelesenen Werten ermittelte Standardabweichung liegt $z$ wischen $\pm 0,0036$ und $\pm 0,0076$ und beträgt 1,16 bis $2,26 \%$ der im Bereich von 0,282 bis 0,328 gefundenen Extinktionen. Die Streuung ist demnach im absteigenden Teil der Absorptionskurve etwa doppelt so groß als im Bereich des Kurvenanstiegs und des -gipfels. - Die gefundene Beziehung zwischen maximaler Extinktion und Kurvenanstieg für alle untersuchten Dihydroxycholansäuregemische ist der $\mathrm{Ab}$ bildung 3 zugrunde gelegt. Auf der Abszisse wurden die jeweiligen maximalen Extinktionen, auf der Ordinate die Differenzen aus der Extinktion des jeweiligen Absorptionsmaximums und der Extinktion bei $375 \mathrm{~m} \mu$ aufgetragen.

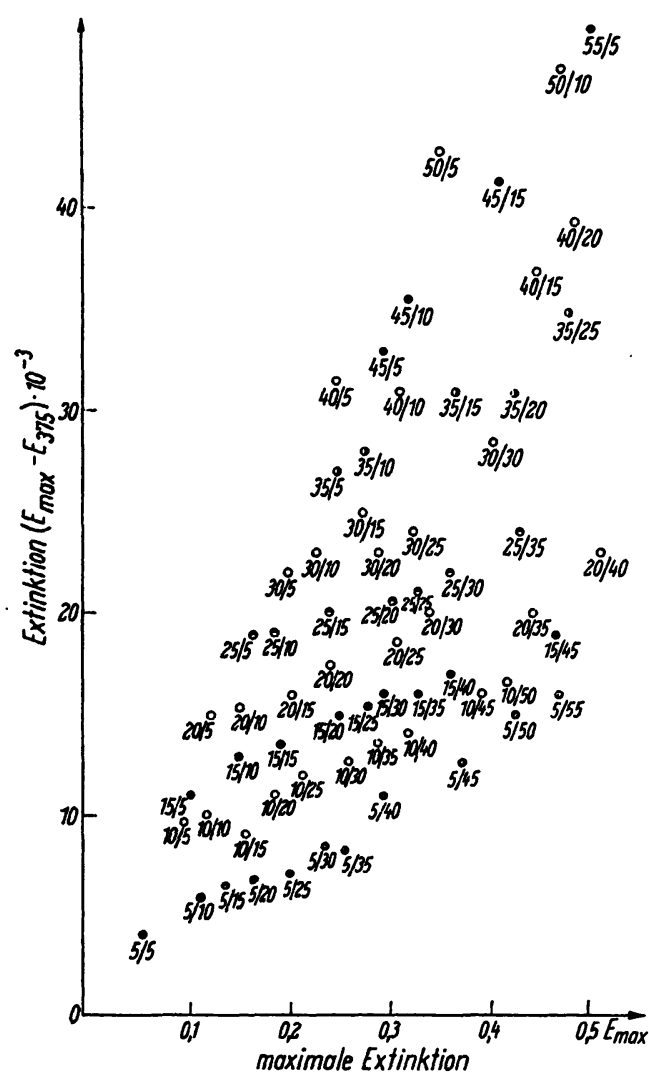

Abb. 3

Beziehung zwischen maximaler Extinktion und der Differenz aus maximaler Extinktion und der Extinktion bei $375 \mathrm{~m} \mu$ für 66 zwischen 5 und $60 \mu \mathrm{g}$ in $5 \mu \mathrm{g}$-Differenzen kombinierten Desoxycholsäure/Chenodesoxycholsäure-Gemische. Die den Punkten beigefügten Zahlen stellen die Desoxycholsäure/Chenodesoxycholsäure-Mengen dar.

Für die einzelnen Gemische ergibt sich demnach eine charakteristische Lage. Die gefundenen Punkte für Gemische mit gleichbleibendem Desoxycholsäure- und zunehmendem Chenodesoxycholsäuregehalt liegen dabei auf Geraden, deren Steilheit mit dem Chenodesoxycholsäuregehalt zusammenhängt. Mit steigendem Desoxycholsäureanteil dagegen nimmt die beschriebene Extinktionsdifferenz zu. Mit Hilfe dieser Darstellung ist es auf Grund der Beziehung zwischen maximaler Extinktion und der Differenz zwischen maximaler Extinktion und der Extinktion bei $375 \mathrm{~m} \mu$ möglich, die Zusammensetzung eines Dihydroxycholansäuregemisches zu bestimmen. Dazu wurden die entsprechenden Kieselgelbezirke der beschriebenen Reaktion mit 65 proz. Schwefelsäure unterworfen und danach die Absorptionskurven zwischen $375 \mathrm{~m} \mu$ und dem Absorptionsmaximum aufgenommen. Die maximale Extinktion wird auf der Abszisse, die Differenz der Extinktionen bei $375 \mathrm{~m} \mu$ und dem Absorptionsmaximum auf der Ordinate aufgesucht. Die in diesen Punkten errichteten Senkrechten schneiden sich in dem Punkt, der die gesuchten Mengen der beiden Dihydroxycholansäuren repräsentiert. Es empfiehlt sich, zur genauen Festlegung des Absorptionsmaximums die Messung der Extinktionen in kleinen Intervallen zwischen 375 und $390 \mathrm{~m} \mu$ durch- 
zuführen. Falls Extinktionswerte außerhalb des von uns angegebenen Bereichs der Maximalextinktionen liegen, ist das Reaktionsgemisch mit 65 proz. Schwefelsäure zu verdünnen. Wir bestimmen zur Analyse eines unbekannten Gemisches $4 \mathrm{mal}$ gleiche aufgetragene Mengen. Da wir unser Verfahren auf $5 \mu \mathrm{g}$ Differenzen eingerichtet haben, ist es weiterhin zweckmäßig, Bestimmungen der doppelten und halben Mengen, eventuell von Dritteln der ursprünglich aufgetragenen Mengen durchzuführen, um Zwischenwerte den bekannten Analysenpunkten zu nähern. Damit lassen sich weitgehend Bestimmungsfehler ausschalten, wenn ungünstige Mischungsverhältnisse (z. B. $2 \mu \mathrm{g}$ Desoxycholsäure $+10 \mu \mathrm{g}$ Chenodesoxycholsäure) vorliegen. Zur Überprüfung unserer Methode isolierten wir nach dem Verfahren von CAREY (7) freie Gallensäuren aus Menschengalle und analysierten die chromatographisch erhaltenen Flecke. Jedem Ansatz wurden in einem zweiten Untersuchungsgang bekannte Mengen freier Gallensäuren auf der Dünnschichtplatte zugesetzt. Die erhaltenen Werte sind aus Tabelle 3 ersichtlich.

Tab. 3

Spektrophotometrische Bestimmung von freien, nach CAREY (7) gewonnenen Gallensäuren ( $D C+C D C)$ aus Menschengallen (Nr. 1, 2, 3) mit und ohne Zusätzen. Werte in $\mu \mathrm{g}$

\begin{tabular}{lrcc}
\hline & ohne Zusatz & $\begin{array}{c}\text { zugesetzte } \\
\text { Menge }\end{array}$ & $\begin{array}{c}\text { gefundene } \\
\text { Menge }\end{array}$ \\
\hline Cholsäure & 6 & 20 & \\
1 & 10 & 10 & 26,5 \\
2 & 18 & 10 & 20 \\
3 & & & \\
Desoxycholsäure & 5 & 10 & 15 \\
1 & 5 & 20 & 25 \\
2 & 10 & 10 & 20 \\
3 & & & \\
Chenodesoxychol- & & & \\
säure & 5 & 20 & 25 \\
1 & 10 & 10 & 20 \\
2 & 15 & 10 & 25 \\
3 & & & \\
\hline
\end{tabular}

\section{Diskussion}

$\mathrm{Zu}$ dem beschriebenen Verfahren, die dünnschichtchromatographisch bisher nur ungenügend $z u$ trennenden beiden Dihydroxycholansäuren durch ihre Absorptionskurven $\mathrm{zu}$ analysieren, wurden wir durch eine Arbeit von Wootron und Wiggins (8) angeregt. Diese Autoren konnten durch Vergleichen zahlreicher von Gemischen bekannter Mengen von Desoxycholsäure und Chenodesoxycholsäure aufgenommener Infrarotspektrogramme mit dem Kurvenbild des zu analysierenden unbekannten Gemisches zu einer annähernd quantitativen Aussage kommen. Das von Gänshirt, Koss und Morianz (1) angegebene Verfahren, die dünnschichtchromatographisch nur un- vollständig zu trennenden Dihydroxycholansäuren als Summe bei enger Spalteinstellung des Spektralphotometers bei $385 \mathrm{~m} \mu$ zu messen, d. h. bei einer Wellenlänge, wo die Extinktionskoeffizienten beider Gallensäuren nahezu identisch sind, trägt der Tatsache keine Rechnung, dāß die Summe der Extinktionen beider Dihydroxycholansäuren keine Rückschlüsse auf die tatsächlichen Anteile von Desoxycholsäure und Chenodesoxycholsäure in dem zu messenden unbekannten Gemisch erlaubt. Eine Vernachlässigung der Desoxycholsäure zugunsten einer quantitativen Bestimmung der Chenodesoxycholsäure allein bei der Wellenlänge des Absorptionsmaximums der Chenodesoxycholsäure, wie das Carey (7) vorschlug unter Hinweis auf Befunde anderer Untersucher, wonach die Desoxycholsäure beim Menschen nur in geringen Mengen vorkommen soll, halten wir nịcht für angängig, da selbst kleine Beimengungen von Desoxycholsäure zu einer Chenodesoxycholsäurelösung deren Absorptionskurve erheblich verändern. So beträgt das Absorptionsmaximum von $60 \mu \mathrm{g}$ allein 0,665 , die Difterenz des Anstiegs $E_{\text {maximum }}$ $\mathrm{E}_{375}=0,010$, während das Absorptionsmaximum von $55 \mu \mathrm{g}$ Chenodesoxycholsäure und $5 \mu \mathrm{g}$ Desoxycholsäure zusammen 0,461 beträgt. In den nach CAREY (7) hergestellten hydrolysierten Extrakten finden wir nach unserem Verfahren der quantitativen Bestimmung beider Dihydroxycholansäuren zwar mehr Chenodesoxycholsäure als Desoxycholsäure, doch sollte diese mit Anteilen von 10,5 und $5 \mu \mathrm{g}$ gegenüber einem Chenodesoxycholsäuregehalt von 15,15 und $5 \mu \mathrm{g}$ nicht vernachlässigt werden.

Betrachtet man die gefundenen Beziehungen zwischen Absorptionsmaximum und der für ein Gemisch von Desoxycholsäure und Chenodesoxycholsäure zusätzlich charakteristischen Differenz zwischen maximaler Extinktion und Extinktion bei $375 \mathrm{~m} \mu$, so fällt auf, daß die definiexten Punkte in den unteren 2 Dritteln der Abbildung annähernd auf Geraden liegen, deren Verläufe durch die Kombination einer bestimmten Menge Desoxycholsäure mit ansteigenden Mengen Chenodesoxycholsäure charakterisiert werden. Im oberen Drittel der Abbildung können wir durch die Meßpunkte teils nach oben, teils nach unten konvexbogige Kurven ziehen. Dieses verschiedene Verhalten in der Verteilung der Meßergebnisse mag seine Ursache in der relativ geringen $\mathrm{Zahl}$ von Messungen im oberen Drittel des Meßbereiches haben (vgl. Abbildung 3). Messungen der Zwischenwerte erlauben evtl. ein Interpolieren der Anteile von Desoxycholsäure und Chenodesoxycholsäure auch in kleinen Bereichen. Wir sahen uns gezwungen, unsere Messungen auf den Bereich zwischen 5-60 $\mu \mathrm{g}$ mit $5 \mu \mathrm{g}$-Stufen zu beschränken. Durch verschiedene Verdünnungen des zu analysierenden Gemisches unbekannter Mengen Desoxycholsäure und Chenodesoxycholsäure läßt sich dieser Nachteil ausgleichen. Angaben über den Fehlerbereich unserer Methode sind nur in den gewählten $5 \mu \mathrm{g}$-Bereichen möglich und deshalb einer statistischen Berechnung nicht zugänglich. Indessen sind die den Messungen zu- 
grunde liegenden Absorptionskurven von Desoxycholsäure- und Chenodesoxycholsäuregemischen gut reproduzierbar, wie auch Wiederauffindungsversuche entsprechender Mengen der beiden Dihydroxycholansäuren die Brauchbarkeit des Verfahrens bestätigen.
Uber die Anwendung dieser Methode an biologischem Material und die Analyse der dünnschichtchromatographisch ebenfalls nicht zu trennenden Dihydroxycholansäurekonjugate des Taurins und des Glycins wird gesondert berichtet.

\title{
Literatur
}

1. Gänshirt, H., F. W. Koss und K. Morianz, ArzneimittelForsch., Aulendorf 10, 943 (1960). - 2. HormanN, A. F., J. Lipid Res. 3, 127 (1962). - 3. FrosCH, B. und H. WAGENER, diese Z., im Druck. - 4. Hamilton, J. G., Gas-Chrom Newsletter 4, 1
(1963). - 5. ENERoTh, P., J. Lipid Res. 4, 11 (1963). - 6. NoRman, A., Acta chem. scand. 7, 1413 (1953). - 7. Carey, B. B., J. Clin. Invest. 37, 1944 (1958). - 8. Wootron, I. D. P. und H. S. WIGGINs, Biochem. J. 55, 292 (1953).
Dr. med. Bruno Frosch

II. Medizinische Klinik und Poliklinik der Freien Universität Berlin

1 Berlin 19, Spandauer Damm 130

\section{Über die Bestimmung von glykolytischen Fermenten beim Krebskranken}

\author{
Von \\ W. Lührs und K. Chrometzka \\ Aus dem Sanatorium „Bergfrieden" - Rottach-Egern der Arbeitsgemeinscbaft für Krebsbekämpfung Sitz Bocbum - \\ Rubrknappscbaft (Chefarzt: Prof. Dr. W. Lübrs)
}

Herrn Professor Warburg zum 80. Geburtstag in dankbarer Verehrung gewidmet

(Der Schriftleitung zugegangen am 22. Juni 1963)

\begin{abstract}
Die heute in der Klinik gebräuchlichen Fermentbestimmungen werden aufgezeigt. Es werderi die Serumfermentwerte bei 144 Krebskranken ausgewertet. Der Effekt einer Behandlung bei Krebskranken kann durch die Fermentwerte objektiviert werden. Als neuestes Ferment wurde die Glutathionreduktase-Aktivität im Serum bestimmt. Bei 303 Patienten, die wegen eines Malignoms operiert und/oder bestrahlt worden waren, wurden die Aldolase, Milchsäuredehydrogenase und die Glutathionreduktase bestimmt. Hierbei zeigte sich beim Vorhandensein von Rezidiven oder Metastasen ein schnelleres Ansprechen der SGR auf diesen pathologischen Befund.
\end{abstract}

\begin{abstract}
The customary enzyme dèterminations currently used in clinical investigation are given. Serum enzyme levels are evaluated for 144 cancer patients. The effect of treatment on cancer patients can be made objective by these enzyme values. Themost recent enzyme test measures serum glutathione reductase. Aldolase, lactic acid dehydrogenase and glutathione reductase were measured in 303 patients who had been operated on and/or irradiated for cancer. SGR showed the most rapid response to relapses or metastases.
\end{abstract}

In zunehmendem Maße werden heute Fermentaktivitäten im Serum zur klinischen Diagnostik benutzit. Neben der Bestimmung der Fermente in Exkreten oder Körperflüssigkeiten hat der Fermentnachweis im Blut an Bedeutung gewonnen. Er kann unter gewissen Umständen ein gutes $\mathrm{Ma} ß$ für das Vorliegen und die Ausbreitung, damit die Progredienz $(1,2)$ bestimmter Tumoren abgeben. Es muß aber betont werden, daß es sich nicht um sogenannte "spezifische Krebsteste" handelt, sondern um Nachweismethoden, die uns bei der Differentialdiagnose helfen, über latente oder progrediente Phasen der Krebskrankheit etwas auszusagen und eine objektive Auswertung der Therapie $(1,2)$ ermöglichen. - Der älteste Fermentnachweis, die Diastasebestimmung im Blut und im Urin ist über 50 Jahre alt. Ebenso ist die Bestimmung der Phosphatasen seit 30 Jahren im klinischen Gebrauch. Für die Klinik ist, auch im Hinblick auf ein karzinomatöses Geschehen die Bestimmung der alkalischen und sauren Phosphatase noch heute wichtig.

Die alkalische Serumphosphatase ist ein Ferment, das aus organischen Verbindungen Phosphorsäure abspaltet. 